\title{
Changes of the F-Wave in the Acute Phase of Permanent Spinal Cord Ischaemic Injury Predict Spinal Cord Function in Animal Models of Rabbits
}

\author{
Yunfei Zhou \\ Xingming Liu \\ Xueyuan Heng \\ Jian Zhang \\ Qimin Song ( $\square$ songqimin-123@163.com ) \\ Chunmei Chen
}

\section{Research}

Keywords: F-wave, Spinal cord ischaemic injury, Reversible injury, Animal models

Posted Date: June 3rd, 2020

DOl: https://doi.org/10.21203/rs.3.rs-31652/v1

License: (c) (1) This work is licensed under a Creative Commons Attribution 4.0 International License. Read Full License 


\section{Abstract}

Background: To explore the changes of the F-wave in the posterior tibial nerve of rabbits after different levels of lumbar spinal cord ischaemic injury and its correlation with motor function and the extent of lumbar spinal cord pathological damage.

Methods: Thirty New Zealand rabbits were randomly divided into 6 groups. The control group $(n=5)$ was used to exclude the influence of anaesthesia and surgery on the F-wave. Different levels of lumbar arteries were ligated in the five experimental groups $(n=5)$. The F-wave was recorded to observe the changes in the acute phase of spinal cord ischaemia.The correlation between the changes of the F-wave in the acute reversible phase and the motor function of the spinal cord was analysed.

Results: The results for the control group indicated that anaesthesia and surgery did not affect the Fwave results. There was no statistically significant difference in the F-wave amplitudes and latency before and after ligation in the 1 and 2 level ligation groups. The F-wave changed immediately after ligation in the 3, 4 and 5 ligation groups. The latency of the F-wave gradually extended, the amplitude of the F-wave gradually reduced.The amplitude variations of the F-wave were positively correlated with the motor function 2 days after ligation, there was a statistically significant difference.

Conclusion: The F-waves in the posterior tibial nerve of rabbits were found to be sensitive to the lumbar spinal cord ischaemic injury and specific to predict motor function.

\section{Background}

Spinal cord injury (SCl) affects many people worldwide and typically has a life-long family and social burden[1]. The New Zealand rabbits are easy to obtain, low cost, and possess mammal-like anatomical structures similar to humans. The use of new Zealand rabbits as an animal model for studying spinal cord ischaemia has allowed us to understand neurophysiological and pathophysiological changes that occur following $\mathrm{SCl}$ and how to prevent and treat SCI[2].The purpose of neurophysiological monitoring is to find ischaemic spinal cord injury in the reversible phase of spinal cord ischaemia. Protective measures can be taken for the prevention of irreversible spinal cord injury during surgery. At present, scholars hold the view that less-than-five-minute spinal cord ischaemia will not cause irreversible spinal cord injury[3]. Data have shown that the amplitude of the F-wave could reflect the function status of the anterior horn cells[4]. The role of the F-wave in predicting the prognosis of one patient after conus medullaris infarct has been reported[5]. Iyori evaluated the degree of spinal cord ischaemic injury in rabbits via the F-wave, which was recorded in the plantar muscle by stimulating the rabbits' posterior tibial nerve[6]. In this study, we established different levels of permanent lumbar spinal cord ischaemic animal models via ligation at different levels of the lumbar arteries between the left renal artery and the arterial bifurcate of rabbits. The characteristics of the F-wave in acute spinal cord ischaemia and its correlation with motor function were analysed, which provides a theoretical basis for protecting spinal cord function by detecting spinal cord injury in the acute reversible phase of spinal cord ischaemic injury. 


\section{Materials And Methods \\ 2.1. Perioperative management}

The present study was carried out in strict accordance with the Use of Laboratory Animals.(National Research Council of USA, 1996). The present study was carried out in strict accordance with Laboratory Animal Regulations of the National Science and Technology Commission[7]. The management of the laboratory animals conformed to the recommendations in the Guide for the Care and Use of Laboratory Animals of the National Institutes of Health. The handling of laboratory animals and their use conformed to the Guidelines for Animal Experiments at Fujian Medical University $₫$ Medical Laboratory Animal Management Regulations. The protocol was approved by the Committee on the Ethics of Animal Experiments of the Union Hospital of Fujian Medical University (Fuzhou, China; permit no. 12-5923). All experimental animals were fed under the same conditions at the institute, where they had access to food and water adlibitum, and were housed individually in metal cages under a $12 \mathrm{~h}$ light/dark cycle at a regulated temperature of $25-26^{\circ} \mathrm{C}$ and a relative humidity of $50-65 \%$. The housing facility maintained national standards, in compliance with the Laboratory Animal-Requirements of Environment and Housing Facilities (GB 14925-2001)[8]. The rabbits in the groups with low Tarlov scores[9]were maintained on the premises with sufficient water and food under standard animal housing conditions. They could feed themselves with the help of an animal administrator. The paralyzed lower limbs were moved with the help of an animal administrator every $2 \mathrm{~h}$.

\subsection{Experimental animals and grouping}

A group of 30 healthy male and female New Zealand White rabbits (17 males and 13 females; weight: $3.0-3.5 \mathrm{~kg}$; age: $11 \pm 1.2$ months) were randomly selected for this experiment. Rabbits were purchased from the Center for Animal Experiments of Hubei Province (Wuhan, China). Rabbits in the experimental group were randomly divided into five groups $₫ n=5 \rrbracket$ according to the different numbers of lumbar arteries being ligatured between the left renal and arterial bifurcate. Group 1 underwent a ligation of the lumbar artery at one level (L1). Group 2 underwent a ligation of the lumbar artery at two levels (L1-L2). Group 3 underwent a ligation of the lumbar artery at three levels (L1-L3). Group 4 underwent a ligation of the lumbar artery at four levels (L1-L4). Additionally, group 5 underwent a ligation of the lumbar artery at five levels (L1-L5). The control group $₫ n=5 \rrbracket$ was used to exclude the effects of anaesthesia and surgery on the F-wave of the posterior tibial nerve. For rabbits in the experimental group, F-waves were recorded continuously within 2 hours after ligation of the lumbar arteries. Then, the changes of the F-wave in the acute stage of spinal cord ischaemia were observed. The percentage values of the postoperative F-wave waveform when the F-wave was steady after ligation and the preoperative baseline were recorded. In the control group, a group of F-waves were recorded every 30 minutes after anaesthesia for 3 hours to exclude the effect of anaesthesia on the F-wave. The control group underwent a surgical operation to separate the lumbar artery without ligation of the lumbar artery. F-waves were recorded at different times before and after surgery to exclude the effect of surgery on the F-wave. All rabbits were sacrificed with 
deep intravenous sodium pentobarbital anaesthesia $(100 \mathrm{mg} / \mathrm{kg}) 2$ days after surgery. Haematoxylin and eosin (HE) staining was performed to observe pathological changes of the spinal cord between L2 and L4.

\subsection{Anaesthesia management}

Anaesthesia in all the rabbits was induced by marginal ear vein administration of a dose of $1 \mathrm{ml} / \mathrm{kg}$ of $3 \%$ pentobarbital sodium (100 mg/kg; China Langchem, Inc., Shanghai, China) and was maintained at $1 / 3-1 / 2$ of the initial dose according to the response of the animals during the experiment. A tracheal tube was inserted and connected to a respirator to control the rabbits'breathing during the experiment; nitrous oxide and oxygen at a 2:1 ratio were inhaled. Another intravenous line of lactated Ringer's solution (Henan Huali Pharmaceutical Co., Ltd., Pingdingshan, China) was infused according to the amount of bleeding. During the experiment, body temperature was monitored continuously with a rectal thermometer and was maintained between 38 and $39^{\circ} \mathrm{C}$ with an electric blanket.

\subsection{Surgical technique}

Skin preparation was applied to the abdomen, the lower extremity ankle and the foot bottom. An alcohol scrub was used to disinfect the area. The limbs were then fixed to the operating table. Additional local anaesthesia, containing $0.5 \%$ lidocaine hydrochloride ( $1 \mathrm{ml}$; Henan Huali Pharmaceutical Co., Ltd.), was applied to the abdominal wall, and a $10 \mathrm{~cm}$ longitudinal incision was made on the abdomen. The abdominal cavity intestines were carefully reversed to the right side, with warm and wet gauzes to reduce the evaporation of water and heat loss. The retroperitoneal area separates the abdominal aorta from the inferior vena cava and isolates all five lumbar arteries between the left renal artery and arterial bifurcation. If there was artery variation, then that animal was excluded. During the course of the experiment, air conditioning was used to keep the room temperature between $24^{\circ} \mathrm{C}$ and $26^{\circ} \mathrm{C}$, and the rectal temperatures of the rabbits were maintained at no less than $38^{\circ} \mathrm{C}$. Due to the amount of intraoperative blood loss, saline infusions were added intravenously. After the surgical procedures, the skin and muscles of the area were sutured.

\subsection{Monitoring technique for the F-wave of the tibial nerve}

Needle-stimulating electrode (Axon Systems, Inc., Hauppage, NY, USA) cathodes were used to stimulate the proximal end of the rabbit ankle joint. The anode, as the reference electrode, was under the stimulus electrode. The stimulating electrodes were connected to an EpochXP-2000 electrical stimulator (Axon Systems, Inc.). The placement of the recording electrode was as follows: the needle-stimulating electrode (Axon Systems, Inc., Hauppage, NY, USA) cathode was in the foot plantar muscle abdomen surface; the anode was in the bottom of the foot plantar muscle abdomen's tendon, with a distance of approximately $5 \mathrm{~mm}$ between the two; and the wire between the stimulus electrode and the recording electrode was 
grounded. The parameter settings were as follows: stimulus intensity at approximately 4-8 $\mathrm{mA}$ (approximately $20 \%$ above the supramaximal stimulus for the $\mathrm{M}$ response); single pulse automatic stimulation; stimulation frequency: $1 \mathrm{~Hz}$; and stimulation interval: $0.3 \mathrm{~ms}$. The recording parameters were as follows: display sensitivity: 500 uv/div; time baseline: $3-5 \mathrm{~ms} /$ div; bandpass filter range: $50-3000 \mathrm{~Hz}$; average processing of 10 signals; signal analysis time: $50 \mathrm{~ms}$; and notch filter: $50 \mathrm{~Hz}$ closed.

\subsection{Statistical analysis}

SPSS17.0 was used for statistical analysis. The measurement data were expressed byx \pm s. The amplitude and latency of the rabbits after anaesthesia and surgery in the control group were used for comparison via repeated measurement of variance analysis. The paired t-test was used on the experimental group before and after ligation. The Pearson correlation test was then used to analyse the relations between the amplitude change of the F-wave and the motor function 2 days after ligation. The test level was set at $\mathrm{a}=$ 0.05 .

\section{Results}

\subsection{Animal model establishment and typical F-wave waveform}

Thirty New Zealand white rabbits were used in this experiment. Two rabbits were excluded due to lumbar artery variation or intraoperative bleeding. The remaining rabbits successfully underwent the surgical procedure and post-operative evaluation process. After anaesthesia, the rabbits were free to eat. The success rate of the animal models was $93.3 \%$. After anaesthesia, all animals obtained ideal waveforms in this group. The latency of the F-wave of all 30 rabbits before ligation was $11.2 \pm 1.4 \mathrm{~ms}$ and the amplitude was $790.3 \pm 589.5 \mu \mathrm{V}$.

\subsection{The control group}

Analysis of Variance of a Single Set of Repeated Measurement Data was used to analyse, after anaesthesia, the amplitude of the control group at different times. The spherical test results were $\chi 2=$ $34.634, \mathrm{P}<0.05$. The data did not meet the Huynh-Feldt condition or satisfy the spherical hypothesis. There was no statistically significant difference in the corrected variance analysis results $(F=0.59$, $P>0.05)$. Analysis of Variance of a Single Set of Repeated Measurement Data was used to analyse, after anaesthesia, the latency of the control group at different times. The spherical test results were $\chi 2=$ $30.761, P<0.05$. The data did not meet the Huynh-Feldt condition or satisfy the spherical hypothesis. There was no statistically significant difference in the corrected variance analysis results $(F=1.738$, $P>0.05)$. Analysis of Variance of a Single Set of Repeated Measurement Data was used to analyse the amplitude of the different times before and after the operation in the control group. The results of the 
spherical test were $\chi 2=6.071 \otimes P>0.05$. The data satisfied the spherical assumptions, and no freedom correction was required. There was no statistically significant difference $(F=1.212, P>0.05)$. Analysis of Variance of a Single Set of Repeated Measurement Data was used to analyse the latency of the different times before and after the operation in the control group. The results of the spherical test were $\chi 2=6.727$, $\mathrm{P}<0.05$. The data did not meet the Huynh-Feldt condition, and the spherical hypothesis was not satisfied. There was no statistically significant difference in the corrected variance analysis results $(F=3.969, P>$ 0.05). Therefore, the changes in the F-wave during the course of the experiment were not related to anaesthesia and surgery.

\subsection{Experimental group}

No waveform changes in the F-waves were found after ligation in group 1 and group 2.The waveform of the F-wave began to change rapidly after lumbar artery ligation in group 3, group 4, and group 5 . The latency began to increase and the amplitude to decrease immediately. The waveforms began to decrease after $0.9 \pm 0.3 \mathrm{~min}, 0.7 \pm 0.3 \mathrm{~min}$, and $0.8 \pm 0.2 \mathrm{~min}$, respectively, in group 3 , group 4 , and group 5 . Then, they started to stabilize after $1.3 \pm 0.3 \mathrm{~min}, 1.9 \pm 1.6 \mathrm{~min}$, and $1.9 \pm 0.5 \mathrm{~min}$, respectively, in group 3 , group 4 , and group 5(Fig ure1).

\subsubsection{Amplitude changes}

In the experimental group, the amplitude change can be recorded to be consistent with the damage degree after spinal cord ischaemia. The F-wave amplitude disappeared in group 5 after lumbar artery ligation. There was no statistically significant difference according to the amplitude paired t-test after lumbar artery ligation when the F-wave was steady compared to before the operation in group 1 and group 2 (all $P>0.05$ ). There was a statistically significant difference according to the amplitude paired $t-$ test after lumbar artery ligation when the F-wave was steady compared to before the operation in group 3 , group 4, and group 5 (all Pख0.05) (Table 1).

\begin{tabular}{|lllll}
\hline group & before surgery(\%) & after surgery $(\%)$ & $P$ & $t$ \\
Group1 & $100.0 \pm 0.0$ & $100.6 \pm 1.6$ & 0.512 & 0.741 \\
Group2 & $100.0 \pm 0.0$ & $98.1 \pm 2.8$ & 0.209 & 1.495 \\
Group3 & $100.0 \pm 0.0$ & $78.1 \pm 9.0 \mathrm{a}$ & 0.005 & 5.472 \\
Group4 & $100.0 \pm 0.0$ & $33.5 \pm 16.6 \mathrm{a}$ & 0.001 & 8.941 \\
Group5 & $100.0 \pm 0.0$ & $0 \pm 0$ & $/$ & $/$ \\
a, paired t-test after and before lumbar artery ligation, $\mathrm{P} \otimes 0.05$. \\
\hline
\end{tabular}

Table 1 
The percentage values of the F-wave amplitude after ligation and the baseline before ligation at different times ( $x \pm s)$

\subsubsection{Latency changes}

There was no statistically significant difference according to the latency paired t-test after lumbar artery ligation when the F-wave was steady compared to before the operation in group 1 and group 2 (all P> 0.05). The latency of the F-wave began to extend rapidly in group 3, group 4 , and group 5 . There was a statistically significant difference according to the paired t-test in the latency after lumbar artery ligation when the F-wave was steady compared to before the operation in group 3 and group 4 (all P凶0.05) (Table 2)

\begin{tabular}{|lllll}
\hline group & before surgery $(\mathrm{ms})$ & latency after surgery $(\mathrm{ms})$ & $P$ t \\
Group1 & $11.0 \pm 1.7$ & $11.0 \pm 1.7$ & 0.215 & 1.567 \\
Group2 & $11.1 \pm 1.4$ & $11.2 \pm 1.5$ & 0.099 & 2.138 \\
Group3 & $12.1 \pm 1.7$ & $12.8 \pm 2.0 \mathrm{a}$ & 0.03 & 3.283 \\
Group4 & $11.4 \pm 1.5$ & $13.7 \pm 1.4 \mathrm{a}$ & 0.001 & 8.061 \\
Group5 & $11.2 \pm 1.3$ & $/$ & $/$ & $/$ \\
a, paired t-test after and before lumbar artery ligation, $P<0.05$. & \\
\hline
\end{tabular}

Table 2

F-wave latency after and before ligation at different times ( $\mathrm{X} \pm \mathrm{s}$ )

\subsection{Histomorphological observation of lumbar spinal cord pathological sections.}

Normal spinal cord tissue with complete structure and neuronal cells with normal morphology in the control group, group 1 and group 2 could be seen under the light microscope. The spinal cord structure was basically complete in group 3. Point foci degeneration, necrosis, and mild edema of the grey matter could be seen under the light microscope. We could see many morphologically normal neurons. Several neurons became smaller, and the cytoplasm became concentrated. The density of the nuclear chromatin increased, showing apoptotic changes. The spinal cord structure basically disappeared in group 5, with obvious congestion and swelling. There were a large number of vacuolar degenerations and a small number of normal neurons. The nuclei of most neurons were atrophic or dissolved; the cytoplasm was eosinophilic; and the Nissl bodies in the cytoplasm disappeared(Figure 2). The experimental results in group 4 was between those in group 3 and group 5 . 


\subsection{Results of spinal motor function scores}

There was no statistically significant difference in motor function score 2 days after ligation according to the two independent samples Wilcoxon rank sum test between the control group and group 1, group 2 , and group 3 (all $\mathrm{P}>0.05$ ). There was no statistically significant difference in motor function score according to the paired-sample Wilcoxon rank sum test between before and 2 days after ligation in group 1 , group 2 , and group 3(all $P>0.05$ ). There were statistically significant differences in motor function score 2 days after ligation according to the two independent samples Wilcoxon rank sum test between the control group and groups 4 and $5(Z=2.835$ and $Z=2.739$, all $P \otimes 0.05)$. There were statistically significant differences in motor function score according to the paired-sample Wilcoxon rank sum test before and 2 days after ligation in group 4 and group $5 \llbracket Z=2.07$ and $Z=2.126$, all $P \bowtie 0.05$ ) (Table 3).

\begin{tabular}{|lcc}
\hline group & before surgery & after surgery 2d \\
Control group & $5.0(5.0,5.0)$ & $5.0(5.0,5.0)$ \\
Group 1 & $5.0(5.0,5.0)$ & $5.0(5.0,5.0)$ \\
Group 2 & $5.0(5.0,5.0)$ & $5.0(5.0,5.0)$ \\
Group 3 & $5.0(5.0,5.0)$ & $5.0(5.0,5.0)$ \\
Group 4 & $5.0(5.0,5.0)$ & $4.0(2.0,5.0) \mathrm{a} \mathrm{b}$ \\
Group 5 & $5.0(5.0,5.0)$ & $0.0(0.0,0.8) \mathrm{a} \mathrm{b}$ \\
a, the two independent samples Wilcoxon rank sum test compared with the control group, $P<0.05$. \\
b, the paired-sample Wilcoxon rank sum test before and 2 days after ligation, $P<0.05$. \\
\hline
\end{tabular}

Table 3

Motor function score (score) M(QL, QU)

\subsection{Correlation analysis}

Based on the Pearson's relation analysis, the percentage value of the F-wave amplitude when the F-wave was steady after ligation and the baseline before ligation was positively correlated with the motor functions $(r=0.964)$. There was a statistically significant difference $(P<0.01)$.

\section{Discussion}

The neurophysiological mechanism of the F-wave is that nerve impulses, being generated after strong stimulation, pass along motor nerve fibres by retrograde conduction to the spinal cord and then to excite motor neurons, which causes excitement[10]. The excitement then spreads along the motor nerve to the muscle[11]. F-waves only measure a small portion of the motor unit pool tested by antidromically activating some of the alpha-motor neurons[12]. The amplitude of the F-wave is the overall excitability 
index of the measurement of the motor neuron pool[13]. F-wave can be used to measure the functional status of the entire motor pathway and to determine its velocity, which is the elapsed time between a stimulus artefact and the first F-wave. Additionally, it is closely correlated with the length of the alpha motor nerve[14].

The posterior $1 / 3$ of the spinal cord is supplied with blood by two continuously posterior spinal arteries[15], whereas the anterior $2 / 3$ is supplied with blood by an intermittent anterior spinal artery[16]. As a result of the good continuity of the posterior spinal artery and many posterior root arteries, the possibility of ischaemia being generated in the posterior spinal artery is very small, but the damage of the root artery mainly has an impact on the anterior $2 / 3$ spinal cord[17]. Ischaemia of the anterior $2 / 3$ spinal cord would affect the spinal neurons in the anterior horn of the spinal cord. The amplitude of the F-wave can reflect the excitement of the spinal anterior horn motor neurons. We established different levels of spinal cord ischaemia animal models, the results indicate that when the injury of the spinal cord ischaemia has developed to a certain extent, the latency of the F-wave starts to extend quickly, and the amplitude starts to reduce quickly and eventually disappears.The results of this experiment further proved that the F-wave is sensitive to spinal cord ischaemia.

The aim of electrophysiological monitoring is to find the injury in the early stage of spinal cord damage. Thus, we should take protective measures to prevent irreversible spinal cord injuries. It is reported that Fwave monitoring during spinal operation offers the opportunity to monitor part of the proximal nervous system in a different manner than the use of the somatosensory evoked potentials or motor evoked potentials[18]. Reports about F-wave monitoring to protect the spinal motor conduction pathway are rare. The results of our experiment indicate that the F-wave is sensitive to spinal cord ischaemic injury and specific to reflecting movement function.

This study has several limitations. First,the spine cord of The New Zealand rabbits are more small than the humanity,may cause more spine cord injury.Second,how to ensure that the silicone column is parallel to the long axis of the spine without distortion or skew? If it is distorted, the experimental results will be inaccurate. Future expansive studies that use mammals more similar to humans and increase MRI examination may help uncover the further underlying mechanisms.

In short, the amplitude of the F-wave is sensitive to ischaemic injury of the spinal cord and can identify ischaemic injury in the reversible period. In addition, amplitude changes of the F-wave can predict the motor function of the spinal cord. All these above features provide evidence that the F-wave can be used to discover injury during the acute reversible period of the spinal cord and lay the basis for taking measures to prevent irreversible spinal cord injuries.

\section{Conclusion}

The F-waves in the posterior tibial nerve of rabbits were found to be sensitive to the lumbar spinal cord ischaemic injury and specific to predict motor function. 


\section{Abbreviations}

Spinal cord injury (SCl)

lumbar vertebrae one (L1)

lumbar vertebrae two (L2)

lumbar vertebrae three (L3)

lumbar vertebrae four (L4)

lumbar vertebrae five (L5)

Haematoxylin and eosin (HE)

\section{Declarations}

\section{Ethics approval and consent to participate:}

"All procedures performed in studies involving animals were in accordance with the ethical standards of the institution or practice at which the studies were conducted.(Fuzhou, China; permit no. 12-5923)"

\section{Consent for publication $\rrbracket$}

Not applicable.

\section{Availability of data and materials $\rrbracket$}

The datasets used and analysed during the current study are available from the corresponding author on reasonable request.

\section{Competing interests:}

The authors declare that they have no competing interests.

\section{Funding:}

The present study was supported by funds from the Health Department of Shandong Province of China (grant nos. 2011QW008 and 2015WS0375). 


\section{Author contributions:}

All authors contributed to the study conception and design. Material preparation, data collection and analysis were performed by $\mathrm{YZ}, \mathrm{XL}, \mathrm{XH}, \mathrm{CC}$,and JZ. The first draft of the manuscript was written by QS. All authors read and approved the final manuscript.

\section{Acknowledgements:}

Not applicable.

\section{References}

1. Kjell J, Olson L.Rat models of spinal cord injury: from pathology to potential therapies. Dis Model Mech.2016; 9 (10):1125-1137. doi:10.1242/dmm.025833

2. Liu NK, Xu XM. Neuroprotection and its molecular mechanism following spinal cord injury. Neural Regen Res.2012; 7 (26):2051-2062. doi:10.3969

3. de Haan P, Kalkman CJ, Meylaerts SA, Lips J, Jacobs MJ.Development of spinal cord ischemia after clamping of noncritical segmental arteries in the pig. Ann Thorac Surg.1999; 68 (4):1278-1284. doi:10.1016/s0003-4975(99)00727-4

4. Sathya GR, Krishnamurthy N, Veliath S, Arulneyam J, Venkatachalam J. F wave index: A diagnostic tool for peripheral neuropathy. Indian J Med Res.2017; 145 (3):353-357. doi:10.4103

5. 1. Alanazy MH. Conus medullaris stroke. Does $F$ wave predict return of ambulation? Neurosciences (Riyadh).2016; 21 (3):260-263. doi:10.17712

6. Iyori K, Kamiya K, Suzuki O, Kojima A, Kubota K, Ishimoto T, Kobayashi M, Shindo S, Tada Y.Identification of the critical spinal arteries with $\mathrm{F}$ wave-polysynaptic response complex monitoring: an experimental study. J Vasc Surg.2001; 34 (2):330-336. doi:10.1067/mva.2001.114816

7. Shi SS, Yang WZ, Chen Y, Chen JP, Tu XK.Propofol reduces inflammatory reaction and ischemic brain damage in cerebral ischemia in rats. Neurochem Res.2014; 39 (5):793-799. doi:10.1007/s11064-014$1272-8$

8. Tu XK, Zhang HB, Shi SS, Liang RS, Wang CH, Chen CM, Yang WZ.5-LOX Inhibitor Zileuton Reduces Inflammatory Reaction and Ischemic Brain Damage Through the Activation of PI3K/Akt Signaling Pathway. Neurochem Res.2016; 41 (10):2779-2787. doi:10.1007/s11064-016-1994-x

9. Gurer B, Kertmen H, Kasim E, Yilmaz ER, Kanat BH, Sargon MF, Arikok AT, Erguder BI, Sekerci Z.Neuroprotective effects of testosterone on ischemia/reperfusion injury of the rabbit spinal cord. Injury.2015; 46 (2):240-248. doi:10.1016/j.injury.2014.11.002

10. Funaba M, Kanchiku T, Imajo Y, Suzuki H, Yoshida Y, Nishida N, Fujimoto K, Taguchi T.Characteristics of C6-7 myelopathy: assessment of clinical symptoms and electrophysiological findings. Spinal Cord.2016; 54 (10):798-803. doi:10.1038/sc.2015.203 
11. Balbi P.Limitations of the F-wave test in monitoring spinal motoneurone excitability. J Physiol.2016; 594 (13):3845. doi:10.1113/jp272442

12. Haavik H, Niazi IK, Jochumsen M, Sherwin D, Flavel S, Turker KS.Impact of Spinal Manipulation on Cortical Drive to Upper and Lower Limb Muscles. Brain Sci.2016; 7 (1). doi:10.3390/brainsci7010002

13. Li X, Fisher M, Rymer WZ, Zhou P.Application of the F-Response for Estimating Motor Unit Number and Amplitude Distribution in Hand Muscles of Stroke Survivors. IEEE Trans Neural Syst Rehabil Eng.2016; 24 (6):674-681. doi:10.1109/tnsre.2015.2453274

14. Alemdar M.Value of F-wave studies on the electrodiagnosis of carpal tunnel syndrome. Neuropsychiatr Dis Treat.2015; 11:2279-2286. doi:10.2147/ndt.S45331

15. Matsubayashi J, Tsuchiya K, Shimizu S, Kitagawa N, Wakabayashi Y, Kuroda M, Sakurai M, Nagao T.Posterior spinal artery syndrome showing marked swelling of the spinal cord: a clinico-pathological study. J Spinal Cord Med.2013; 36 (1):31-35. doi:10.1179/2045772312y.0000000017

16. Muller KI, Steffensen LH, Johnsen SH.Thrombolysis in anterior spinal artery syndrome. BMJ Case Rep.2012. doi:10.1136/bcr-2012-006862

17. Alshareef M, Krishna V, Ferdous J, Alshareef A, Kindy M, Kolachalama VB, Shazly T.Effect of spinal cord compression on local vascular blood flow and perfusion capacity. PloS one.2014; 9 (9):e108820. doi:10.1371/journal.pone.0108820

18. Stecker MM.A review of intraoperative monitoring for spinal surgery. Surg Neurol Int.2012; 3 (Suppl 3):S174-187. doi:10.4103/2152-7806.98579

\section{Figures}



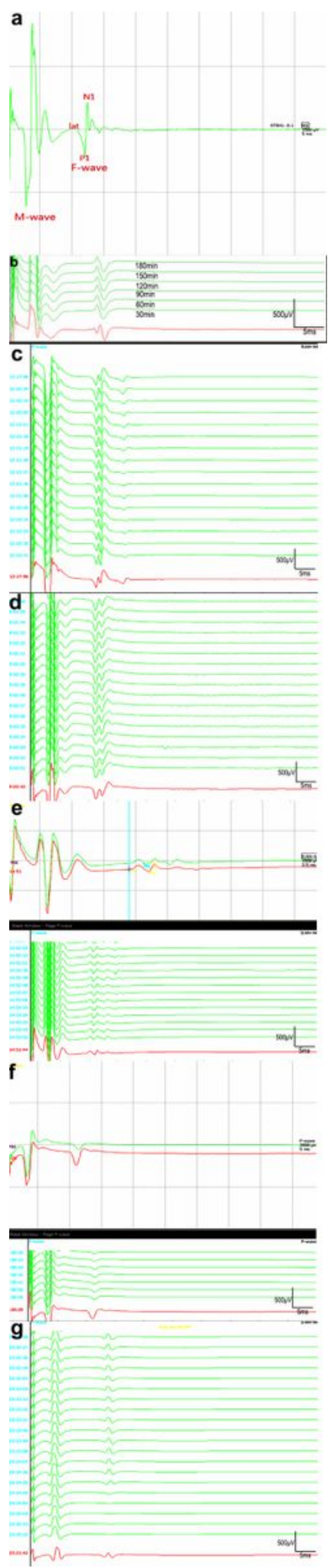

Figure 1

A F-wave of control group and group1-5 The amplitude and latency of F-wave were stable at different times after anaesthesia in the different groups. a A typical F-wave. b Sham group. c Group 1. d Group 2. e Group 3. f Group 4. g Group 5. F-wave latency(Lat) is defined as the duration, in millisecond, from stimulation to the first progressive negative deflection. F-wave amplitude is defined as peak-to-peak amplitude in microvolts (N1-P1). N1 is the first upward negative wave; P1 is the first downward positive 
wave; The red graphics indicate the positive and negative baseline waveform before ligation;The green graphics indicate the positive and negative baseline waveform after lumbar artery ligation. With the increase of the number of lumbar arteries being ligatured, ischemia is further aggravated, which is manifested by the prolongation of the F- wave latency of the posterior tibial nerve and the decrease of the amplitude.

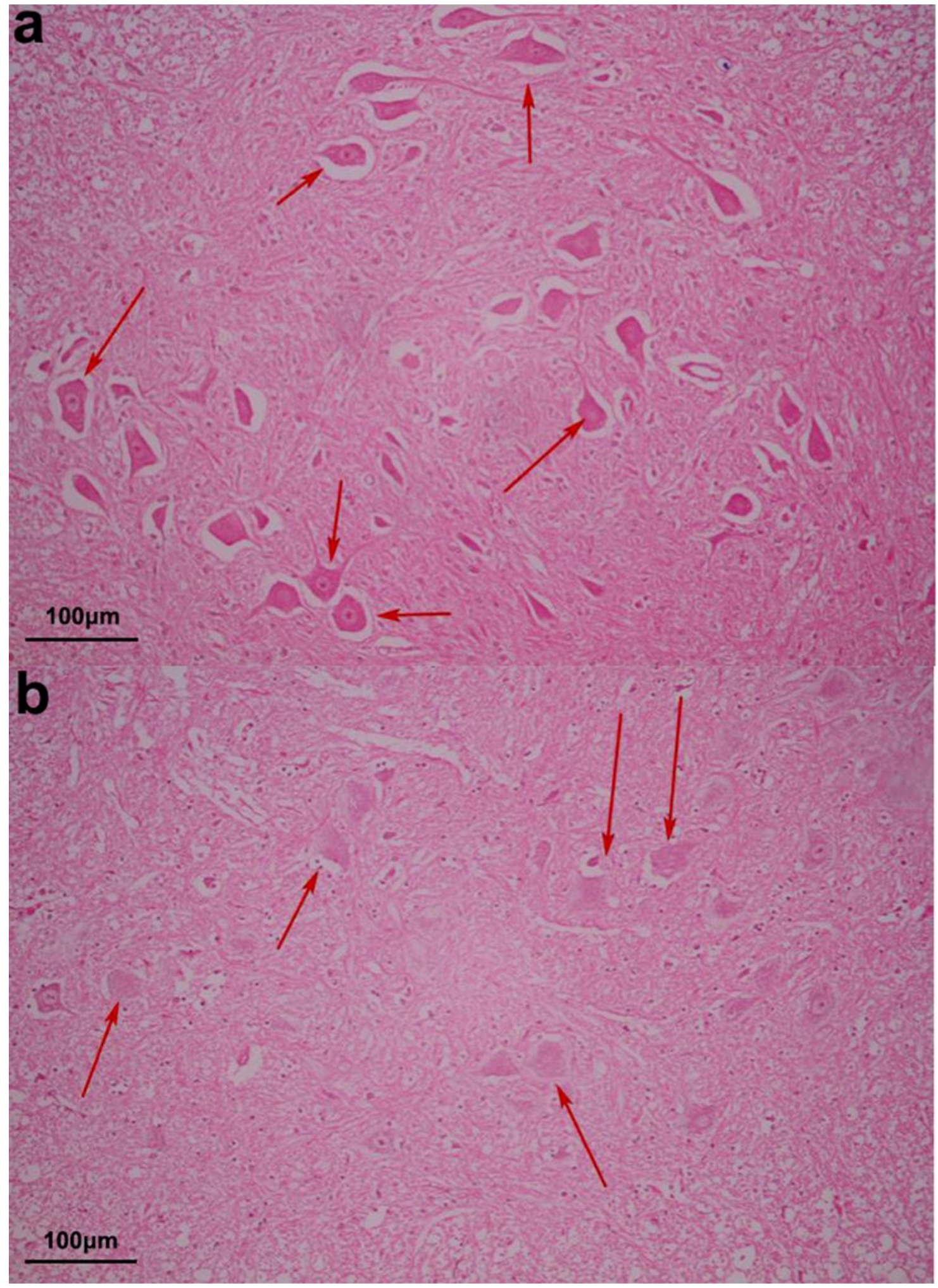

\section{Figure 2}


Haematoxylin-eosin staining of spinal cord sections at the 2 days after operation. a:There are no sign of neurnal damage was observed in the sham group and Intact motor neurons were observed in the ventral horn (indicated by arrows). Magnification, x200;b:Apoptotic and necrotic motoneurons in the ventral horn were observed in group 5(indicated by arrows). Magnification, x200. 